\title{
Bistability and nonequilibrium condensation in a driven-dissipative Josephson array: a c-field model
}

\author{
Matthew Reeves ( $\nabla$ m.reeves@uq.edu.au ) \\ University of Queensland https://orcid.org/0000-0001-8534-8564 \\ Matthew Davis \\ University of Queensland https://orcid.org/0000-0001-8337-0784
}

\section{Article}

Keywords: Nonequilibrium Quantum Systems, Driven-dissipative Josephson Junction Chain, Atomic Bose-Einstein Condensates, C-field Theory

Posted Date: March 3rd, 2021

DOl: https://doi.org/10.21203/rs.3.rs-265517/v1

License: (1) This work is licensed under a Creative Commons Attribution 4.0 International License. Read Full License 


\title{
Bistability and nonequilibrium condensation in a driven-dissipative Josephson array: a c-field model
}

\author{
Matthew T. Reeves* and Matthew J. Davis \\ Australian Research Council Centre of Excellence in Future Low-Energy Electronics Technologies, \\ School of Mathematics and Physics, University of Queensland, St Lucia, QLD 4072, Australia.
} (Dated: January 22, 2021)

\begin{abstract}
Developing theoretical models for nonequilibrium quantum systems poses significant challenges. Here we develop and study a multimode model of a driven-dissipative Josephson junction chain of atomic Bose-Einstein condensates, as realised in the experiment of Labouvie et al. [Phys. Rev. Lett. 116, 235302 (2016)]. The model is based on c-field theory, a beyond-mean-field approach to Bose-Einstein condensates that incorporates fluctuations due to finite temperature and dissipation. We find the c-field model is capable of capturing all key features of the nonequilibrium phase diagram, including bistability and a critical slowing down in the lower branch of the bistable region. Our model is closely related to the so-called Lugiato-Lefever equation, and thus establishes new connections between nonequilibrium dynamics of ultracold atoms with nonlinear optics, exciton-polariton superfluids, and driven damped sine-Gordon systems.
\end{abstract}

\section{INTRODUCTION}

Dissipation is oftenundesirable in the study of quantum systems, as it typically leads to the loss of quantum features such as coherence, entanglement and the washing out of interference. However, in recent years it has been realized that by adding controlled sources of dissipation to many-body quantum systems one can engineer novel quantum states of matter [1-6]. For example, an appropriate environment coupling can be harnessed to generate robust entangled states [7-9], or enable dissipative quantum computation protocols [10]. Further, the addition of driving allows the controlled study of quantum transport processes [11], $\mathcal{P} \mathcal{T}$-symmetric quantum mechanics [12,13], and dissipative phase transitions [14]. The ability to introduce engineered driving and dissipation offers the controlled study of nonequilibrium steady-states, which can exhibit emergent and exotic properties that cannot be achieved at or near equilibrium $[15,16]$. Ultracold atomic gases, simultaneously offering accurate and precise experimental control and tractable theoretical models, offer an excellent platform for exploring nonequilibrium phenomena in quantum systems [17-24].

In this paper we develop a tractable theoretical model to describe the driven-dissipative Josephson array as considered in the experiment of Labouvie et al. [4]. This prototypical nonequilibrium quantum system is shown schematically in Fig. 1(a). The system consists of a long chain of coupled, twodimensional (2D) Bose Einstein condensates (BECs), tightly confined in $z$ and harmonically confined in the $x-y$ plane. Atom losses at a controllable rate $\gamma$ are introduced to a single site (the system) by a tightly-focussed electron beam. The resulting decrease in the system's chemical potential causes an influx of particles from the two neighbouring lattice sites (the reservoir), yielding an effective driving of the system proportional to the nearest-neighbour tunnelling rate $J$. The tunnelling mechanism is filling dependent [Fig. 1(b)], as the reservoir chemical potential $\mu_{R}$ is much larger than the radial

\footnotetext{
*m.reeves@uq.edu.au
}
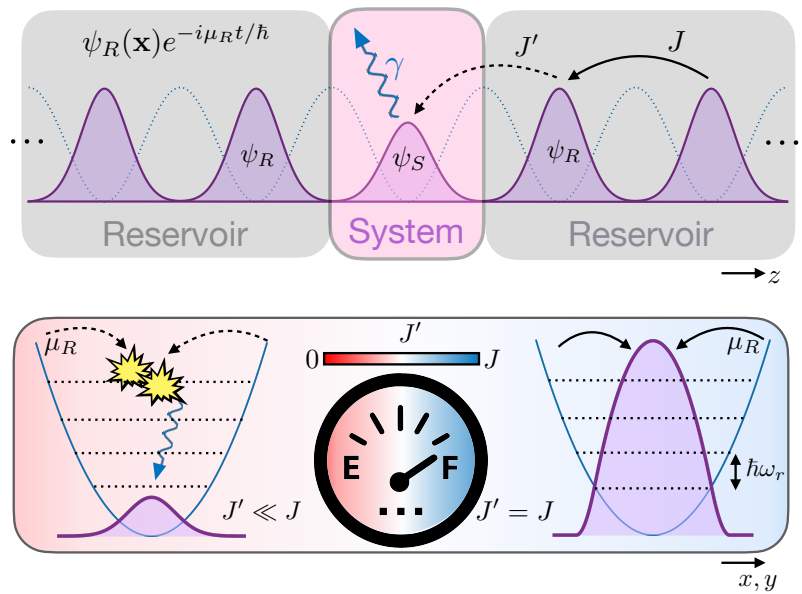

(b)

FIG. 1. (a) The driven-dissipative Josephson junction array is comprised of a prolate atomic BEC loaded into a 1D optical lattice. Dissipation at a rate $\gamma$ is introduced to a single site by a focussed electron beam, and refilling is provided by the neighouring sites. We describe the dynamics of the condensate at the lossy (system) site $\psi_{S}(\mathbf{x}, t)$ by treating the remaining sites as a particle reservoir that provides an AC driving term of spatial profile $\psi_{R}(\mathbf{x})$ and frequency $\mu_{R} / \hbar$. (b) The effective driving $J^{\prime}=\eta J$ depends on the bare tunnelling $J$ of the neighbouring Wannier sites in $z$, but also the overlap $\eta \propto\left\langle\psi_{S} \mid \psi_{R}\right\rangle$ between the radial wave functions of the system and reservoir. The chemical potential of a full site $\mu_{R}$ is much larger than the radial harmonic oscillator spacing $\hbar \omega_{r}$; for a full site (right), particles may resonantly tunnel directly into the condensate, whereas for a nearly depleted site (left) particles instead must tunnel into an excited state and the population of the condensate may only increase via collisional relaxation.

ground state energy of the system, $\hbar \omega_{r}$. When the system site is near full, particles may resonantly couple from the reservoir to the condensate. However, when the system site is depleted, particles tunneling from the neighbouring reservoir sites are too energetic to directly access the system condensate; they must instead tunnel into an excited radial mode resonant with their energy. The physics of the system site is therefore fundamentally a multimode problem. 
The primary result of the experiment was that within a range of dissipation rates $\gamma$ the system exhibited bistability the steady-state atom number of the system site depended on whether it was initially full or empty. The upper (full) branch, which exhibited high particle current associated with superfluid transport from the reservoir, could be partially explained by a mean-field, tight binding model [4, 24-26], while the steady-state filling in the lower (empty) branch could be explained by an effective single particle model with decoherence and a particle-dependent driving rate $[4,11]$. While the basic features of each branch could therefore be understood, a cohesive theoretical description of the system within a single model is currently lacking, and several interesting observations remain unexplained. In particular, in the lower branch of the bistability region a critical slowing down was observed, suggesting the occurrence of a nonequilibrium condensation phenomenon [6, 17, 27] possibly akin to exciton-polariton condensation $[4,28]$. Additionally, the critical dissipation observed in the superfluid phase was considerably smaller than was predicted by the simple single-mode theory [4].

Here we develop a comprehensive model for the system within the framework of c-field theory and numerical explore its features. We describe the dynamics of the system site in terms of a driven-dissipative stochastic Gross-Pitaevskii equation, which is closely related to the Lugiato-Lefever equation of nonlinear optics $[29,30]$. By using a "coherent reservoir model" whereby the reservoirs are described by an AC driving term, we find the c-field model is capable of capturing the bistability and the critical slowing down in the lower branch, and produces a similar nonequilibrium phase diagram to Labouvie et al. [4]. We then investigate a "dynamical reservoir model", incorporating both the effects of finite temperature, as well as the back-action of the system on the reservoirs. This leads to some decoherence between the system and the reservoir, and in doing so we find that the model is capable of quantitatively reproducing the phase boundaries of the nonequilibrium phase diagram.

The outline of the paper is as follows. In Sec. II we introduce the c-field formalism for the study of the drivendissipative site. In Sec. III we reduce our model to a singlemode approximation, and compare analytical results obtained against a tight-binding approach. Section IV presents numerical simulations of our coherent reservoir model, and Sec. V presents our dynamical reservoir model that include backaction and heating effects on the driving sites. Section VI presents discussion and suggestions for future work, before we conclude in Sec. VII.

\section{C-FIELD MODEL}

A natural framework for developing a nonequilibrium multimode model of the system site is provided by c-field theory, also known as the truncated Wigner approximation (TWA) for the interacting Bose gas [31]. Briefly, a master equation for the dynamics of the low-energy, classical modes of the system can be transformed into an equation of motion for the system's Wigner function. Neglecting third-order and higher derivatives - justified at short times or when the number of particles in the system is large - leads to a Fokker-Planck equation, which can then be simulated by an ensemble of trajectories that are solutions to a stochastic differential equation. This approach has been successfully applied to the simulation of the dynamics of Bose-Einstein condensates in a wide range of circumstances [32].

In this work the system of interest is the lattice site where there are atom losses caused a focussed electron beam. The system is described by a classical field $\psi_{S}(\mathbf{x}, t)$, and it has an equation of motion that is a damped and driven stochastic projected Gross-Pitaevskii equation

$$
i \hbar d \psi_{S}=\left\{(\mathcal{L}-i \gamma / 2) \psi_{S}+\mathcal{F}\right\} d t+d W .
$$

Here $\mathcal{L}$ is the projected Gross-Pitaevskii operator

$$
\mathcal{L} \psi_{S} \equiv \mathcal{P}\left\{\left[-\frac{\hbar \nabla^{2}}{2 m}+V(\mathbf{x})+g_{2}\left|\psi_{S}\right|^{2}\right] \psi_{S}\right\},
$$

and the external potential is a symmetric harmonic trap of the form

$$
V(\mathbf{x})=\frac{1}{2} m \omega_{r}^{2}\left(x^{2}+y^{2}\right) .
$$

The effective 2D interaction parameter is given by $g_{2}=$ $g \int d z|w(z)|^{4}$, where $g=4 \pi \hbar^{2} a_{s} / m$ for s-wave scattering length $a_{s}$, and $w(z)$ is the Wannier function associated with the optical lattice in the $z$-direction [33]. The atom loss rate is $\gamma$, and $\mathcal{F}$ is the driving term associated with the reservoir sites. The projection operator $\mathcal{P}$ confines the evolution to the c-field region

$$
\mathcal{P}\{f(\mathbf{x})\}=\sum_{n \in C} \varphi_{n}(\mathbf{x}) \int \mathrm{d}^{2} \mathbf{x}^{\prime} \varphi_{n}^{*}\left(\mathbf{x}^{\prime}\right) f\left(\mathbf{x}^{\prime}\right),
$$

where $C$ contains all modes with energies less than a prescribed energy cutoff; here this amounts to $n_{x}+n_{y} \leq n_{\text {cut }}$, where $n_{x}, n_{y}$ are the quantum numbers of the Cartesian basis states of a symmetric $2 \mathrm{D}$ harmonic oscillator. We assume the condensate is "optically thin" in the z-dimension, i.e., that the intensity of the electron beam does not diminish along the propagation axis, such that the loss rate $\gamma$ can be treated as spatially independent. The Weiner noise term $d W$ associated with the dissipation therefore satisfies

$$
\left\langle d W^{*}(\mathbf{x}, t) d W\left(\mathbf{x}^{\prime}, t^{\prime}\right)\right\rangle=\gamma \delta_{C}\left(\mathbf{x}, \mathbf{x}^{\prime}\right) \delta\left(t-t^{\prime}\right) d t,
$$

where $\delta_{c}\left(\mathbf{x}, \mathbf{x}^{\prime}\right) \equiv \sum_{n \in C} \varphi_{n}(\mathbf{x}) \varphi_{n}\left(\mathbf{x}^{\prime}\right)$ is the kernel of the coherent region projection operator [31]; it behaves as a Dirac delta function when acting on the projected subspace.

The last remaining ingredient is the forcing function $\mathcal{F}$, which physically is provided by the neighbouring sites in the lattice. The experiment of Labouvie et al. [4] spanned approximately $\sim 60$ individual sites. Instead of simulating the entire system, we begin with a simple approximation; we treat the two neighbouring sites as undepleted reservoirs at zero temperature. The driving function $\mathcal{F}$ is thus described by a field which is an equilibrium solution to the Gross-Pitaevskii equation with spatial profile $\psi_{R}(\mathbf{x})$ and chemical potential $\mu_{R}$. The 
forcing hence takes the form of a spatially dependent coherent AC driving term (cf. Fig. 1)

$$
\mathcal{F}(\mathbf{x}, t)=-2 J \psi_{R}(\mathbf{x}) e^{-i \mu_{R} t / \hbar},
$$

where the factor of two comes from the fact that the system has two nearest neighbours. In principle there could be a relative phase between the two driving sites - this would result in the replacement $2 \rightarrow\left(1+e^{i \varphi}\right)$ in Eq. (6), but at this level of approximation this would only result in reduced effective value of $|J|$. We therefore ignore this possibility for the moment, but will consider it further in $\mathrm{Sec} \mathrm{V}$.

For an optical lattice with height $V_{0}$, the bare tunnelling rate $J$ is related to the matrix elements of neighbouring Wannier functions via [33]

$$
J=\int \mathrm{d} z w^{*}(z) \hat{H} w(z-a)
$$

where $\hat{H}=-\hbar^{2} \partial_{z}^{2} / 2 m+V_{0} \sin ^{2}(2 \pi z / \lambda)$ and $a=\lambda / 2$ is the lattice spacing. The effective rate of tunnelling however depends on the overlap between the radial wave functions of the system and reservoir at a given instant in time. From Eq. (1) it follows that the mean system atom number $N_{S}$ evolves according to

$$
d N_{S} / d t=\hbar^{-1}\left(4 \eta J \sqrt{N_{R} N_{S}}-\gamma N_{S}\right),
$$

where $N_{R}$ is the reservoir atom number, and $\eta$ is a so-called Frank-Condon factor given by

$$
\eta(t)=\frac{\operatorname{Im}\left\{\left\langle\psi_{R} \mid \psi_{S}(t)\right\rangle\right\}}{\sqrt{N_{R} N_{S}}}, \quad|\eta| \leq 1,
$$

such that in the steady state $N_{S} / N_{R}=(4 J \eta / \gamma)^{2}$. Note that $\eta$ is inherently built into the c-field model presented here, whereas in Labouvie et al. [4] this factor was added phenomenologically and assumed to be linear in the atom number difference.

The driving reservoir field $\psi_{R}(\mathbf{x})$ is determined by solving for the ground state of the projected GPE by integrating the damped projected GPE

$$
i \hbar \partial_{t} \psi_{R}=(1-i \gamma)\left(\mathcal{L}-\mu_{R}\right) \psi_{R}=0 .
$$

A given value of the reservoir chemical potential $\mu_{R}$ hence self-consistently determines both the forcing profile $J \psi_{R}(\mathbf{x})$, and driving frequency $\mu_{R} / \hbar$.

\section{A. Relation to Lugiato-Lefever model}

The model we have presented for the driven-dissipative BEC Eq. (1) is closely related to the the Lugiato-Lefever equation (LLE) [29, 30], which is usually expressed in the form

$$
\partial_{t} \psi=-i \alpha \nabla^{2} \psi-i \beta|\psi|^{2} \psi-(\gamma+i \Omega) \psi-\delta .
$$

The LLE is widely used in the setting of nonlinear optics, and can also be derived in the small amplitude limit from the ACdriven damped sine-Gordon equation $[34,35]$. As such the
LLE can describe a variety of systems, including coupled pendula, extended Josephson junctions, easy-axis ferromagnets in microwave fields, rf-driven plasmas, whispering gallery mode resonators, and chemical reaction-diffusion systems (see, e.g., Refs. [35, 36] and references therein). Our system corresponds to the case of anomalous dispersion $\alpha<0$, defocussing nonlinearity $\beta>0$, and blue-detuned driving $\Omega>0$; however, all of these may in general be of either sign [35] depending on the physical system. The LLE and generalized versions also arise in the context of superfluid excitonpolariton systems with coherent pumping [28, 37-42].

\section{SINGLE-MODE APPROXIMATION}

Before solving the full SPGPE (1), let us first consider the mean-field treatment when the system consists of a single mode. We will find that this approximation provides a qualitative understanding of the bistability and hysteresis observed in Labouvie et al. [4]. Furthermore, analytical results can be derived for this model and these will provide a useful point for comparison with the numerical analysis of the subsequent sections.

We assume that the mode shape for the system can be approximated by the ground state of the 2D harmonic trap, such that $\psi_{S}(x, y, t) \approx c_{S}(t) \phi_{0}(x) \phi_{0}(y)$ and $\mathcal{L} \psi_{S}=\hbar \omega_{r} \psi_{S}$. In the mean-field approximation the noise term can be neglected, and Eq. (1) reduces to

$$
i \hbar \frac{d c_{S}}{d t}=\hbar \omega_{r} c_{S}+g_{0}\left|c_{S}\right|^{2} c_{S}-2 J c_{R}-i \frac{\gamma}{2} c_{S},
$$

where $g_{0}=g_{2} / 2 \pi \ell_{h o}^{2} \equiv g_{2}\left(\int d x\left|\phi_{0}(x)\right|^{4}\right)^{2}$, and $\ell_{h o}=$ $\sqrt{\hbar / m \omega_{r}}$. Eliminating the $\hbar \omega_{r}$ term by moving to a rotating frame, $c_{S} \rightarrow c_{S} e^{i \omega_{r} t}$, the driving term takes the form

$$
c_{R}=\sqrt{n_{R}} e^{-i \Delta t},
$$

where $\hbar \Delta=\mu_{R}-\hbar \omega_{r} \geq 0$ is the detuning, and

$$
c_{S}=\sqrt{n_{S}} e^{-i(\omega t-\phi)} .
$$

where $n_{S}, \omega$ and $\phi$ are to be determined. For a steady state solution, the system must be locked to the reservoir driving frequency, with $\omega=\Delta=$ const., giving the two conditions

$$
\frac{n_{S}}{n_{R}}=\left(\frac{4 J \sin \phi}{\gamma}\right)^{2}, \quad \frac{n_{S}}{n_{R}}=\left(\frac{2 J \cos \phi}{g_{0} n_{S}-\hbar \Delta}\right)^{2} .
$$

Eliminating $\phi$ then yields the following cubic equation for the density

$$
n_{S}\left[\left(g_{0} n_{S}-\hbar \Delta\right)^{2}+\gamma^{2} / 4\right]=4 J^{2} n_{R}
$$

Real and positive roots of Eq. (16) define steady-state solutions, phase locked to the drive, with a constant phase lag $\phi$ given by

$$
\tan \phi=\frac{\gamma / 2}{g_{0} n_{S}-\hbar \Delta}+\pi \Theta\left(\hbar \Delta-g_{0} n_{S}\right)
$$



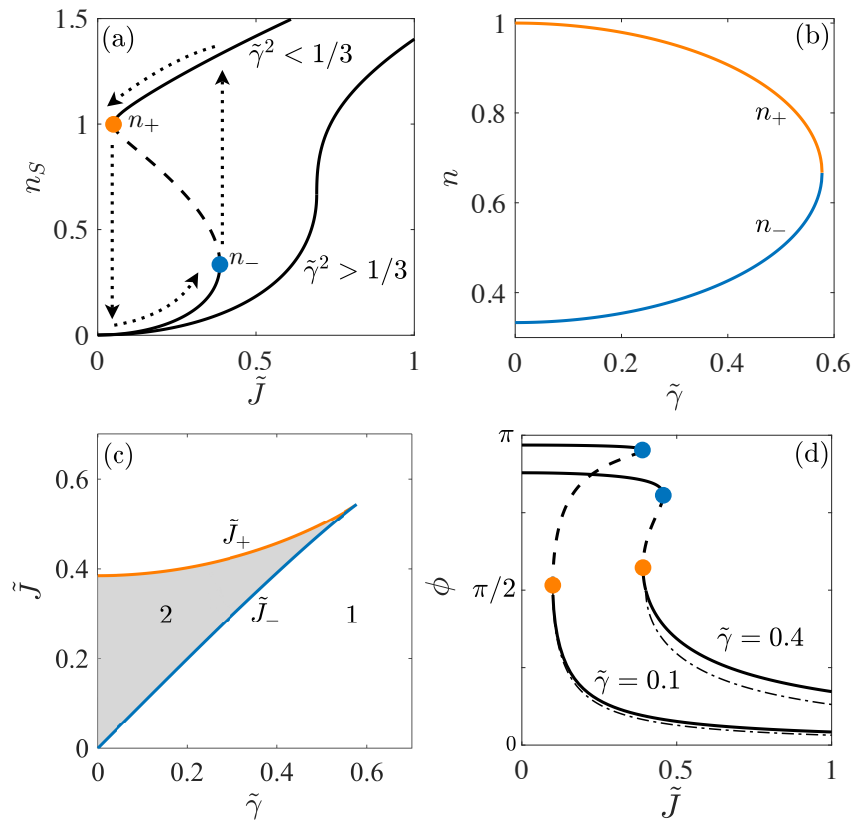

FIG. 2. Bistability of the single-mode model. (a) Examples of $n_{S}$ vs. $\tilde{J}$ curves in the bistable $\left(\tilde{\gamma}^{2} \leq 1 / 3\right)$ and monostable regions $\left(\tilde{\gamma}^{2}>1 / 3\right)$ of parameter space. The dashed region indicates the unstable solution, and the circle markers indicate the critical points $\left(\tilde{J}_{-}, n_{+}\right)$and $\left(\tilde{J}_{+}, n_{-}\right)$. (b) Occupation number vs. $\tilde{\gamma}$ for the critical points $n_{+}$and $n_{-}$. (c) Bistability phase diagram in the $\tilde{\gamma}-\tilde{J}$ plane. The shaded region exhibits bistability (two stable solutions) whereas the unshaded regions contains only one solution. (d) Examples of the phase lag $\phi$ against $\tilde{J}$ for large and small dissipation values where bistability is observed. As in (a), the dashed line indicates the unstable region, and circle markers show the critical points. The dash-dot lines show the Josephson model prediction for the upper branch [Eq. (24)] for comparison (see text).

The second term involving the Heaviside function $\Theta(x)$ arises as Eq. (15) only defines $\phi$ up to a shift of $\pi$; this ensures $\phi$ varies continuously on the interval $[0, \pi]$.

It is convenient to define $\Delta^{-1}$ and $\hbar \Delta$ as the natural units of time and energy respectively, reducing Eq. (16) to the dimensionless form

$$
n_{S}^{3}-2 n_{S}^{2}+\left(1+\tilde{\gamma}^{2}\right) n_{S}=\tilde{J}^{2}
$$

where $\tilde{\gamma}=\gamma / 2 \hbar \Delta$ and $\tilde{J}=\sqrt{n_{R}} 2 J / \hbar \Delta$. For convenience we have also absorbed the nonlinearity coefficient $g_{0} / \hbar \Delta$ via the substitution $\left\{c_{S}, c_{R}\right\} \rightarrow \sqrt{g_{0} / \hbar \Delta}\left\{c_{S}, c_{R}\right\}$ such that $n_{R}=1$.

Equation (18) can now be analysed using standard methods for cubic equations. It permits either one or three solutions depending on the values of $\tilde{\gamma}$ and $\tilde{J}$, see Fig. 2(a). The region where multiple solutions exist is bounded by the critical points; the density at these critical points, $n_{S}=n_{ \pm}$[ see Fig. 2(a), left curve] can be found by differentiating Eq. (18) and solving the resulting quadratic. This yields

$$
n_{ \pm}(\tilde{\gamma})=\frac{2 \pm \sqrt{1-3 \tilde{\gamma}^{2}}}{3},
$$

as graphed in Fig. 2(b). When $\tilde{\gamma}^{2}>1 / 3$ Eq. (19) no longer yields real solutions, indicating that the derivative of Eq. (18) is everywhere positive, and hence there is only one solution [Fig. 2 (a), right curve]. For $\tilde{\gamma}^{2}<1 / 3$, multiple solutions emerge via a pitchfork bifurcation [38]; there is either one or three solutions depending on the value of $\tilde{J}$ [Fig. 2 (a), left curve]. Inserting Eq. (19) into Eq. (18) gives the range of $\tilde{J}$ that supports multiple solutions, $\tilde{J}_{-} \leq \tilde{J} \leq \tilde{J}_{+}$, where

$$
\tilde{J}_{ \pm}^{2}(\tilde{\gamma})=\frac{2}{27}\left[1+9 \tilde{\gamma}^{2} \pm\left(1-3 \tilde{\gamma}^{2}\right)^{3 / 2}\right]
$$

as shown in Fig. 2(c). To assess the number of stable solutions, we write $c_{S} \rightarrow c_{S}+\delta c e^{-i \lambda t}$, with $c_{S}$ given by Eqs. (14), (16) and (17). Retaining terms linear in $\delta c$ yields

$$
\lambda=-i \tilde{\gamma} \pm \sqrt{\left(n_{S}-1\right)\left(3 n_{S}-1\right)}
$$

giving stable solutions whenever $\operatorname{Im}\{\lambda\} \leq 0$, i.e.,

$$
\operatorname{Im}\left\{\sqrt{\left(n_{S}-1\right)\left(3 n_{S}-1\right)}\right\} \leq \tilde{\gamma} .
$$

This condition is satisfied for $n_{S} \leq n_{-}$or $n_{S} \geq n_{+}$as per Eq. (19). The steady-state populations can hence be separated into a lower branch $n_{S} \leq n_{-}$, a middle branch $n_{-}<n_{S}<n_{+}$, and an upper branch $n_{S} \geq n_{+}$. The upper and lower branches are stable and the central branch is unstable, i.e., the system is bistable, and exhibits a hysteresis cycle as shown in Fig 2(a). The shaded region bounded by $J_{ \pm}$as shown in Fig. 2(c) thus defines the bistable region of the $\gamma$ - $J$ plane.

Mathematically the above results are identical to those obtained for a single mode dispersive optical cavity with cubic nonlinearity [38, 43]. Although in the above analysis we have neglected the effects of noise, the single mode system can in fact be solved exactly, with the inclusion of fluctuations, within the generalized $P$ representation [43]. As discussed in Refs [38, 43], retaining fluctuations in the single-mode model does not dramatically change the mean-field stability diagram; rather the essential difference is that steady-state filling becomes bimodal rather than truly bistable - the system tends to explore the vicinity near the stable mean field solutions on shorter timescales, and stochastically switches between the two at longer timescales. The inclusion of noise therefore does not drastically alter the qualitative behaviour from the mean-field predictions, suggesting that multimode effects are essential to capture the system behaviour observed in the experiment of Labouvie et al. [4].

As Eq. (12) is a rather drastic simplification of the system under consideration, it is useful to compare the above results with the Josephson model considered in Labouvie et al. [4]. This approach instead considers an infinite array of coupled, single-mode sites

$$
i \hbar \frac{d c_{n}}{d t}=-J\left(c_{n+1}+c_{n-1}\right)+g_{0}\left|c_{n}\right|^{2} c_{n}-i \frac{\gamma}{2} c_{n} \delta_{m n} .
$$

In addition to the trivial steady state $c_{n}=0 \forall n$, this model supports a steady solution in which all sites have identical filling, and a relative phase difference $\phi \equiv\left|\phi_{n}-\phi_{n+1}\right|$ between neighbouring sites given by

$$
\sin \phi=\frac{\gamma}{4 J}
$$




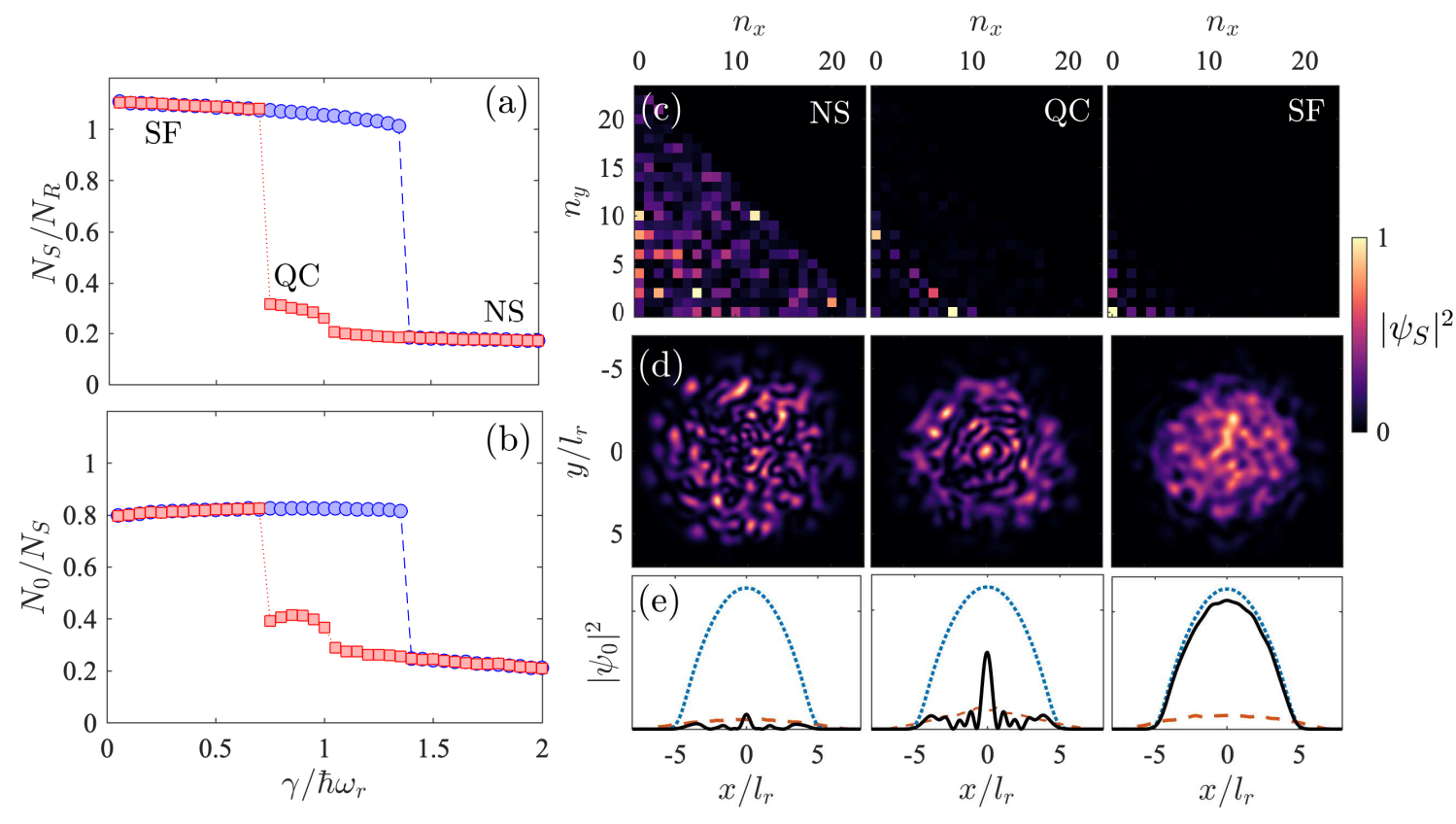

FIG. 3. (a) Relative filling $N_{S} / N_{R}$, and (b) condensate fraction $N_{0} / N_{S}$ vs. dissipation $\gamma$ for at a fixed driving strength $J / \hbar \omega_{r}=0.35$. Blue circle and red square markers indicate the resulting steady state from beginning with full and empty initial conditions respectively (see text). $(\mathrm{c}, \mathrm{d})$ : Examples of the instantaneous particle density $\left|\psi_{S}\right|^{2}$ for the normal state (NS), quasicondensate (QC) and superfluid (SF) phases, in (c) mode space, and (d) position space. (e) Slices along the $x$-axis at $y=0$, showing the condensate density $\left|\psi_{0}\right|^{2}$ (black solid), final time-averaged noncondensate density (red dashed), and reservoir density (blue dotted).

Clearly, this model predicts a breakdown of superflow at the critical value $\gamma_{c}=4 J$, since Eq. (24) has no solution for $\gamma>$ $\gamma_{c}$.

Whereas the Josephson model only supports identical filling across all sites, the solutions to Eq. (16) can exhibit an "overfilling" $\left(n_{S} / n_{R}>1\right)$ under strong driving [Fig. 2(a)]. This overfilling is unphysical in the context of the driven damped lattice system we consider here (no such behaviour was observed in Labouvie et al. [4]), and (as we will show in Sec. V) is a consequence of neglecting the back-action of the system on the reservoir. However, here we are primarily interested in the bistability region, where this effect is less pronounced than for large values of the driving. Further, comparing Eqs. (15) and (24) shows the phase lag differs by a factor of $\sqrt{n_{S} / n_{R}}$. This discrepancy also turns out to be small in the vicinity of the bistability region; the predicted phase lag curves are in semi-quantitative agreement, especially for smaller values of dissipation, see Fig. 2(d).

Finally, note that both models yield very similar predictions for the critical dissipation strength of the superfluid branch: expanding Eq. (20) to first order in $\tilde{\gamma}$ yields $\tilde{J}_{-} \approx \tilde{\gamma}$, which is equivalent to $\gamma_{c}=4 \mathrm{~J}$ as predicted in the Josephson model see Fig 2(c).

From the preceeding analysis, it is clear that the drivendamped single mode model captures the qualitative behaviour of the bistability. However, it does not capture many of the other key findings of Labouvie et al. [4]. In particular, the bistability region boundaries differ in shape to those observed in the experiment, and further the solutions of Eq. (12) show no indication of critical slowing down within the bistable re- gion. In the following section we thus turn to solving the full multimode model.

\section{MULTIMODE COHERENT RESERVOIR MODEL}

\section{A. Numerical Implementation}

We now proceed with a full numerical treatment of the driven damped SPGPE. Following the standard procedure for c-field implementations, Eq. (1) is numerically integrated via a pseudospectral method with quadrature (formally equivalent to the Galerkin method [31, 44]). The basis is a standard Cartesian Hermite-Gauss representation, which diagonalizes the single-particle Hamiltonian and thus approximately diagonalizes the nonlinear problem when the mode occupation is low [45]. The nonlinear term is treated using the appropriate Gaussian quadrature rules [31], such that the matrix elements are implemented exactly for all modes in the c-field region. The simulations were performed using XMDS2 [46]. Throughout we work in units of the radial harmonic oscillator, i.e., length, time and energy are expressed in units of $l_{r}=\sqrt{\hbar / m \omega_{r}}, \omega_{r}^{-1}$ and $\hbar \omega_{r}$ respectively.

As usual with c-field implementations, some quantities (such as total energy and particle number) inevitably exhibit a dependence on the chosen energy cutoff. Some care is therefore required in both choosing the energy cutoff, and interpretation of results. To verify that our results did not depend on the choice of cutoff, we calculated the condensate number 

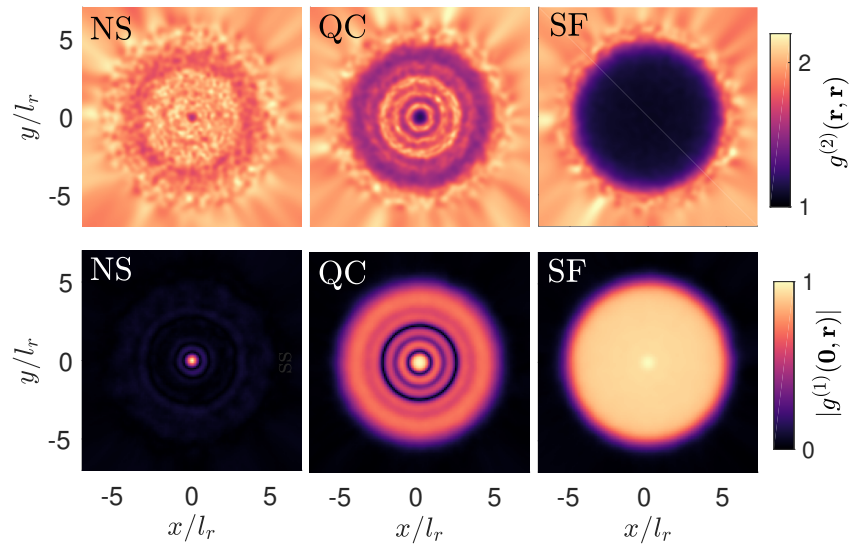

FIG. 4. First- and second-order correlation functions $g^{(2)}(\mathbf{r}, \mathbf{r})$ (top row) and $g^{(1)}(\mathbf{0}, \mathbf{r})$ (bottom row) for the three phases shown in Fig. 3. Left column: normal state. Middle column: quasicondensate. Right column: superfluid.

$N_{0}$, as determined from the largest eigenvalue of the one-body density matrix $\rho\left(\mathbf{r}, \mathbf{r}^{\prime}\right)$. Within our c-field framework this is readily constructed from a time-average of the steady state as

$$
\rho\left(\mathbf{r}, \mathbf{r}^{\prime}\right)=N_{t}^{-1} \sum_{n=1}^{N_{t}} \psi_{S}^{*}\left(\mathbf{r}, t_{n}\right) \psi_{S}\left(\mathbf{r}^{\prime}, t_{n}\right) .
$$

Varying the cutoff in the range of $\sim 2 \mu_{R}$ to $3 \mu_{R}$, we found that $N_{0}$ was insensitive to the choice in cutoff (variations were on the order of 5\%). The values for $\gamma$ and $J$ at which we observed the key qualitative changes in the system behaviour were also not sensitive to the choice of cutoff, and nor were the qualitative trends observed in the particle number and condensate fraction. Our results for the nonequilibrium phase boundaries can thus be viewed as quantitative predictions, whereas the condensate fraction and particle number only indicate qualitative trends. Unless otherwise specified, we used the dimensionless interaction parameter $C_{n l}=g_{2} / \hbar \omega_{r} l_{r}^{2}=0.2$ and $\mu_{R} / \hbar \omega_{r}=12$, giving $N_{R} \approx 2200$ atoms in the driving reservoir. The cutoff in mode space is set to $n_{\text {cut }}=2 \mu_{R} / \hbar \omega_{r}(=24)$.

\section{B. Nonequilibrium Steady States}

As a driven-dissipative nonlinear system, the steady states of our model are in general expected to be dependent on the initial conditions. Following Labouvie et al. [4], we therefore compare the results of beginning with an initially phase coherent "full" site, against an initially incoherent "empty" site. For the full site the initial condition is the same as the reservoir wave function, $\psi_{S}(\mathbf{x}, t=0)=\psi_{R}(\mathbf{x})$, whereas the empty site has each single particle mode seeded with complex Gaussian noise, scaled such that the initial relative filling $N_{S} / N_{R}$ is $5 \%$. Figure 3 shows the steady state properties of these two cases as $\gamma$ is varied, for a fixed driving strength of $J / \hbar \omega_{r}=0.35$.

Despite the inclusion of noise multimode collisions, the full branch [blue circles] exhibits little difference from the singlemode model discussed in Sec. III. Below the expected dis-
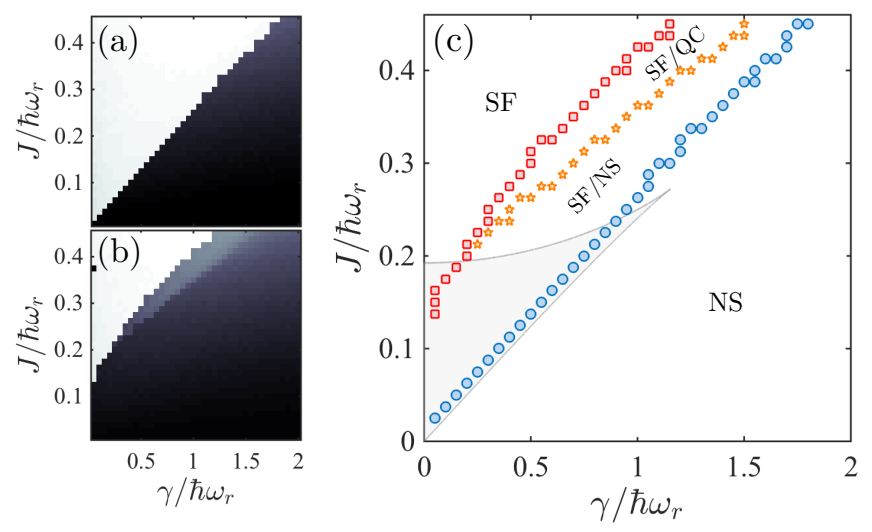

FIG. 5. Steady-state condensate fractions $N_{0} / N_{S}$ in the (a) full and (b) empty branches in the $J-\gamma$ plane. (c) The phase diagram determined by numerically extracting phase boundaries from (a) and (b). The upper and lower boundaries were extracted by detecting the boundary where the condensate fraction exceeded 0.7 , and the middle boundary shows where the it exceeded 0.4 . For comparison the shaded region shows the bistable region of the single-mode system [c.f. Fig. 2(c)].

sipation threshold $\gamma_{c} \sim 4 J$, the system is able to remain in the superfluid phase (SF), and retains approximately unit relative filling [Fig. 3(a)] and condensate fraction $N_{0} / N_{S} \sim 80 \%$ [Fig. 3(b)]. For dissipation above the expected threshold the system eventually becomes depleted, and the steady state phase is an incoherent 'normal state' (NS), containing only a residual relative filling and condensate fraction of $\sim 20 \%$ [Fig. 3(a,b)]. The reduction in condensate fraction in both cases occurs due to the driving noise associated with the dissipation.

In the normal state the particle density is dominated by thermal-like fluctuations, as can be seen in the instantaneous density profiles, [Fig. 3(c,d), left column], and the condensate and noncondensate fraction profiles in [Fig. 3(e), right]. In contrast, in the superfluid state the density closely resembles the profile of the reservoir condensate [Fig. 3(c,d) right]. The the condensate mode profile [Fig. 3(e), right] also closely resembles the (equilibrium) reservoir profile, but is depleted by a small noncondensate fraction.

The "empty" branch [Fig. 3(a,b), red squares] however exhibits a feature not seen in the from the single-mode prediction; the filling and condensate fraction now exhibit an intermediate plateau in the middle of the bistable region (notice this plateau is not observed in the full branch [blue circles]). This region exhibits $\sim 30 \%$ relative filling [Fig. 3(a)], $40 \%$ condensate fraction [Fig. 3(b)] and reduced fluctuations in the density profile [Fig. 3(d) and movie in the Supplemental Material [47]]. In this nonequilibrium "quasicondensate" phase (QC) the majority of the particles occupy a band of harmonic oscillator modes that are resonant with the driving frequency [Fig. 3(c) middle]. This same band of states determines the condensate mode, leading to the patterned condensate mode profile in Fig 3(e,middle). For the case $\mu_{R} / \hbar \omega_{r}=12$ shown here, the majority of the particles occupy the band of single- 

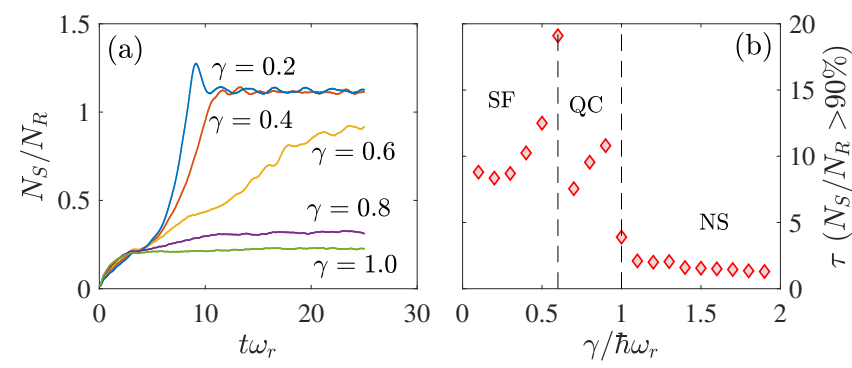

FIG. 6. Critical slowing down observed at short timescales, similar to Labouvie et al. [4]. Data from 40 trajectories for each $\gamma$. (a) Filling vs. time at fixed $J / \hbar \omega_{r}=0.35$ for a range of $\gamma$. (b) Refilling time $\tau$, measured as the time taken for the relative filling to exceed $90 \%$ within the empty branch.

particle modes satisfying $n_{x}+n_{y} \sim 8$. The detuning from $\mu_{R}$ is a combination of the zero-point energy $\hbar \omega_{R}$ and a blueshift due to the repulsive interactions.

To gain further insight into the three nonequilibrium steadystates, in Fig. 4 we measure the spatial phase coherence via the first-order correlation function

$$
g^{(1)}\left(\mathbf{r}, \mathbf{r}^{\prime}\right)=\frac{\left\langle\psi_{S}^{*}(\mathbf{r}, t) \psi_{S}\left(\mathbf{r}^{\prime}, t\right)\right\rangle}{\left[\left\langle\left|\psi_{S}(\mathbf{r}, t)\right|^{2}\right\rangle\left\langle\left|\psi_{S}\left(\mathbf{r}^{\prime}, t\right)\right|^{2}\right\rangle\right]^{1 / 2}},
$$

and the density coherence via the second-order correlation function

$$
g^{(2)}\left(\mathbf{r}, \mathbf{r}^{\prime}\right)=\frac{\left\langle\psi_{S}^{*}(\mathbf{r}, t) \psi_{S}^{*}\left(\mathbf{r}^{\prime}, t\right) \psi_{S}(\mathbf{r}, t) \psi_{S}\left(\mathbf{r}^{\prime}, t\right)\right\rangle}{\left\langle\left|\psi_{S}(\mathbf{r}, t)\right|^{2}\right\rangle\left\langle\left|\psi_{S}\left(\mathbf{r}^{\prime}, t\right)\right|^{2}\right\rangle},
$$

where the averages are computed as time averages in the steady state as in Eq. (25). As can be seen in Fig. 4, the correlations in the normal phase and the superfluid phase of the nonequilibrium steady-states are similar to that expected at thermal equilibrium. The normal state almost completely lacks phase coherence (rapid decay of $g^{(1)}(\mathbf{0}, \mathbf{r})$ ), and $g^{(2)}(\mathbf{r}, \mathbf{r}) \sim 2$ throughout the system, as is typical for a thermal state [48]. Similarly the superfluid phase exhibits almost complete phase and density coherence throughout the system with $g^{(1)}(\mathbf{0}, \mathbf{r}) \sim 1$, and $g^{(2)}(\mathbf{r}, \mathbf{r}) \sim 1$, as is the case for a condensate well below the critical temperature [48].

The quasicondensate phase is less typical, and exhibits a partial phase and density coherence; density and phase fluctuations are strong at intermediate radii, but less pronounced near the origin and near the edge of the cloud. The reduced coherence at intermediate radii appears to be due to a proliferation of quantized vortices, as can be seen in Fig. 3(d) (middle) and the movies provided of the dynamics of filling in the three phases in the supplemental material [47].

An overview of the system's steady state behaviour is presented in Fig. 5, which shows the condensate fractions for the full and empty branches within the $\gamma-J$ plane [Fig. 5(a) and (b) respectively], and the corresponding nonequilibrium phase diagram extracted from the boundaries [Fig. 5(c)]. The nonequilbrium phase diagram in Fig. 5 is divided into four regions: in the top left region, the steady state of the system is the superfluid state (SF), regardless of the initial conditions,
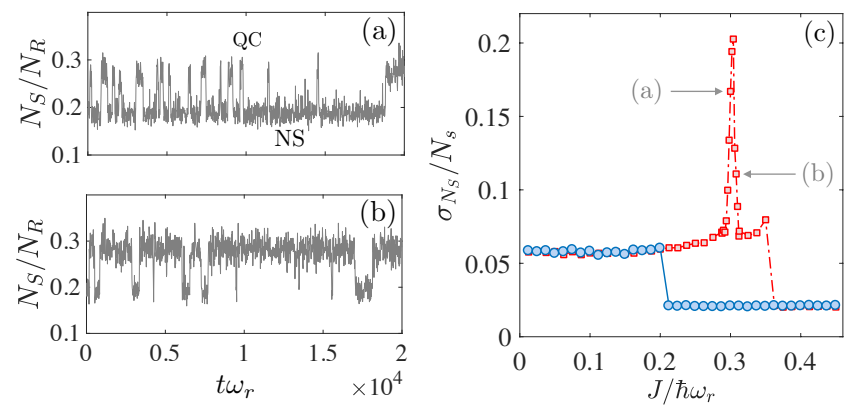

FIG. 7. Critical slowing down on long timescales, due to atom number fluctuations in the steady state. (a,b) Examples of the filling as a function of time for driving just below and above the transition. (c) Standard deviation of the filling number as a fraction of the mean filling number, $\sigma_{N_{S}} / N_{S}$ vs. $J$, for $\gamma / \hbar \omega_{r}=0.75$. Blue circles: full initial conditions, red squares: empty initial conditions. The corresponding values of $J$ for (a) and (b) are also indicated.

while in the bottom right the system ends up in the incoherent normal state (NS). The quasicondensate boundary (orange stars) further separates the bistability region into two distinct regions; above the boundary, the bistability is between the superfluid and quasicondensate phases (SF/QC), while below it is between the superfluid and normal phases (SF/NS).

As in the single-mode model, for an initially full site the phase transition boundary is linear in the $J-\gamma$ plane [Fig. 5(a,c)]. The boundary is practically indistinguishable from the Josephson model prediction $J_{c}=\gamma / 4$, with a linear line of best fit $J_{c}=m \gamma+b$ yielding $m=0.248 \pm 0.003$ and $b=0.015$.

The changes to the lower branch, however, have greatly enlarged the bistability window [Fig 2(c)]; for most values of $\gamma$, the driving $J$ required to enter the superfluid phase is significantly higher than the single-mode prediction, and the upper boundary has changed from a convex shape to slightly concave. Notice that unlike the single mode prediction, phase boundaries no longer "close", at least over the range of $J$ and $\gamma$ considered. While these boundaries may merge at higher values of $J$ and $\gamma$, such values would not likely be accessible in a cold atom experiment and were therefore not considered in this work.

The quasicondensate phase boundary, which traverses diagonally across the bistability region, only appears at sufficiently large $\mu_{R}$ - for example, this state was observed for $\mu_{R} / \hbar \omega_{r}=7$ but not for $\mu_{R} / \hbar \omega_{r} \sim 3$. For $\mu_{R} / \hbar \omega_{r} \sim 3$ the bistability region quite closely resembled the predictions of the single-mode approximation (gray shaded region in [Fig 2 (c)]). In contrast to the other two boundaries, which are sharp transitions, the quasicondensate transition is a smooth crossover, which becomes broader at larger $\gamma$.

\section{Critical Slowing Down}

In Labouvie et al. [4] a critical slowing down was observed within the bistability region, which suggestively co- 
incides with our observation of the quasicondensate phase. In Fig. 6(a) we show the dynamics of the relative filling at experimentally relevant timescales $t \omega_{r} \sim 10$, along with the timescale of the filling in Fig. 6(b) (defined to be when the relative filling exceeds 90\%). We instead observe evidence for slowing down at both phase boundaries bistable region. However, the observed timescales of the slowing down are broadly consistent with Labouvie et al.[4], as is the qualitative shape of the refilling time vs. $\gamma$.

An additional signature of critical slowing down at the quasicondensate transition occurs on much longer timescales; here large atom number fluctuations are observed, as shown in Fig 7. As in the quantum theory of optical bistability [38, 43], the noise allows for a stochastic switching between the normal and quasicondensate phases within the vicinity of the transition [Fig. 7(a,b)], resulting in a clear spike in the fluctuations near the transition [Fig. 7(c)]. At larger $\gamma$ where the transition broadens, the fluctuation peak becomes broader and less pronounced. While the noise is capable of switching the system between the normal and quasicondensate phases, which are relatively "nearby" in terms of atom number, the noise is not large enough to cause switching between the normal and superfluid phase, and therefore no such peak is observed at the other transition points. No indication of switching between normal and superfluid phases was observed in any of the simulations, for integration timescales up to $t \omega_{r} \sim 10^{4}$.

\section{MULTIMODE DYNAMICAL RESERVOIR MODEL}

In the previous section we have shown that the full c-field treatment of empty branch exhibits several differences from the single-mode model, while the full branch exhibits little difference. In particular, it does not resolve the discrepancy between the normal-superfluid boundary as predicted by the Josephson model, $\gamma_{c}=4 J$, with that observed in the experiment of Labouvie et al. of $\gamma_{c} \approx J$ [4]. This suggests that additional physics needs to be incorporated into the model. One key approximation of the previous section was that the neighbouring reservoir sites were undepletable and at zero temperature.

In this section we extend the model to incorporate the effects of back-action of the system site on the reservoir sites, as well as the effects of finite temperature. As shown schematically in Fig. 8, we simulate the dynamics of the c-field for the condensates on the left and right sides of the system site, $\psi_{L}$ and $\psi_{R}$ respectively. Their dynamics are coupled $\psi_{S}$ to incorporate the backaction of the lossy site.

The system's dynamics are still governed by Eq. (1), but the forcing now takes the form

$$
\mathcal{F}(\mathbf{x}, t)=-J\left[\psi_{L}(\mathbf{x}, t)+\psi_{R}(\mathbf{x}, t)\right]
$$

which replaces Eq. (6). The refilling of the driving condensates by the rest of the lattice is mimicked by coupling $\psi_{L}$ and $\psi_{R}$ to a thermal bath [see Fig. 8]. The equation of motion for the reservoir sites is thus taken to be the simple-growth SPGPE [48] with the addition of back-action coupling from

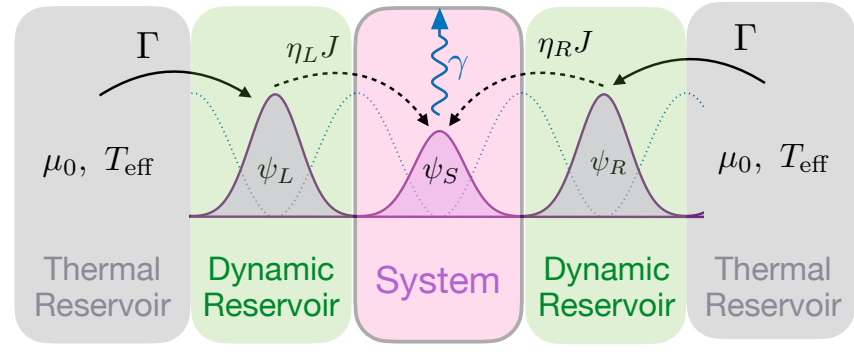

FIG. 8. A schematic of the dynamical reservoir model, governed by Eq. (1), and Eqs. (28)-(30). The system is now driven by two independent, finite temperature driving condensates described by $\psi_{L}(\mathbf{x}, t)$ and $\psi_{R}(\mathbf{x}, t)$, whose dynamics are governed by Eq. (29). The refilling of the driving condensates from the rest of the lattice is mimicked by coupling them to a thermal bath at effective temperature $T_{\text {eff }}$ and chemical potential $\mu_{0}$.

the system field $\psi_{S}$ :

$$
i \hbar d \psi_{j}=\left\{(1-i \Gamma)\left(\mathcal{L}\left[\psi_{j}\right]-\mu_{0}\right) \psi_{j}-J \psi_{S}\right\} d t+d \zeta_{j}
$$

Here $j=\{L, R\}$ and the noise correlations are

$$
\left\langle d \zeta_{j}(\mathbf{x}, t) d \zeta_{j}^{*}\left(\mathbf{x}^{\prime}, t^{\prime}\right)\right\rangle=\left(2 \Gamma k_{B} T_{\mathrm{eff}}\right) \delta_{c}\left(\mathbf{x}, \mathbf{x}^{\prime}\right) \delta\left(t-t^{\prime}\right) d t,
$$

where $T_{\text {eff }}$ is the effective temperature, $\mu_{0}$ is the chemical potential of the reservoirs and $k_{B}$ is Boltzmann's constant. The introduction of the effective temperature parameter $T_{\text {eff }}$ serves to model the effects of reduced coherence due to a noncondensate fraction and the resulting dephasing of different sites in the lattice. As we are using a c-field model with an energy basis cutoff, the essential observable of interest is the resulting reduced condensate fraction of the system and reservoirs, rather than the value of the temperature itself.

Under Eq. (29), the atom number on the driving sites evolves according to

$$
\frac{d N_{j}}{d t}=\frac{2}{\hbar}\left\{\Gamma\left[\mu_{0}-\bar{\mu}(t)\right] N_{j}-J \eta_{j} \sqrt{N_{j} N_{S}}\right\},
$$

where $\eta$ is the Franck-Condon factor from Eq. (9), and

$$
\bar{\mu}(t)=\frac{1}{N_{j}} \int \mathrm{d}^{2} \mathbf{x}\left(\psi_{j}^{*} \mathcal{L} \psi_{j}+\psi_{j} \mathcal{L} \psi_{j}^{*}\right)
$$

is the instantaneous chemical potential of the system. The parameter $\Gamma$ thus accounts for the rate of refilling of the reservoir sites, which physically would come from the tunnelling from neighbouring lattice sites. The refilling rate depends on the instantaneous atom number and deviations of the system from its zero temperature equilibrium through the term $\left[\mu_{0}-\bar{\mu}(t)\right]$. While it is therefore not entirely straightforward to determine the most appropriate choice for $\Gamma$, it is clear that it should be the same order of magnitude as $J / \hbar \omega_{r}$ to mimic the refilling from the rest of the lattice. For the simulation results that we present we have chosen $\Gamma=J / 2 \hbar \omega_{r}$. We found that the results did not depend on $\Gamma$ provided it was proportional to $J$ and was of a comparable magnitude. 
In our simulations in this section we keep the reservoir chemical potential at $\mu_{R}=12 \hbar \omega_{r}$ as in the previous section, but increase the effective temperature. This fixes the total number of atoms at the reservoir sites, while decreasing the condensed fraction. Figure 9 shows filling measures for the dynamical reservoir model as a function of $\gamma$ at fixed $J$ with an effective temperature of $k_{B} T_{\text {eff }} / \hbar \omega_{r}=30$. This results in a condensate fraction of approximately $45 \%$ in the reservoirs when $J=0$. Figure 9(a) shows that the addition of back-action has indeed reduced the robustness of the superfluid phase, with the collapse now occurring at $\gamma_{c} \approx J=0.3$.

Further, the incorporation of a dynamical model for the reservoir sites has alleviated the unphysical "overfilling" previously observed for $\gamma \ll J$ [cf. Fig. 3(a)]; $N_{S} / N_{R}$ now only marginally exceeds unity at small dissipation. In our dynamical reservoir model, the quasicondensate phase appears only as a transient state at intermediate times, collapsing to the normal state at later times. The plateau associated with the quasicondensate phase is therefore absent in the relative filling curve, which is determined from the steady-state behaviour. However, the intermediate time window where the quasicondensate is observed is similar to the experimentally relevant timescales $\sim 10-30 \mathrm{~ms}$, suggesting this phase is potentially experimentally relevant even though it is no longer stable at long times.

An interesting feature of Fig. 9(b) is that when the system is in the superfluid phase, the condensate fraction of the system site actually increases with increasing $\gamma$, at the expense of the condensate fraction of the reservoirs. A possible explanation for this outcome is that the coherent condensate atoms tunnel more easily into the system from the reservoirs than the thermal atoms, thus reducing the coherence of the reservoirs and increasing that of the system. Once the system collapses into the normal phase, the filling and condensate fractions of the reservoirs recover to near their equilibrium values.

The first- and second order correlation functions for the dynamical reservoir model were qualitiatively unchanged from those presented for the coherent reservoir model in Fig. 4. However, the bistability phase diagram resulting from this model has some significant differences, As shown in Fig. 10, for the choice of $k_{B} T_{\text {eff }} / \hbar \omega_{r}=30$ the transition boundary of the upper branch is now in quantitative agreement with Labouvie et al. [4], with $\gamma_{c} \approx J$. The boundary of the lower branch is also quantitatively consistent with the observations of Labouvie et al. [4], being well-described by a power law fit with an exponent close to $1 / 2$ as expected for an incoherent hopping process. We find that the effective temperature parameter essentially controls the slope of the transition boundary of the upper branch, as depicted in the inset of Fig. 10(c). Thus it seems that finite temperature and dephasing effects are potentially responsible for the observation of $\gamma_{c} \approx J$ by Labouvie et al. [4].
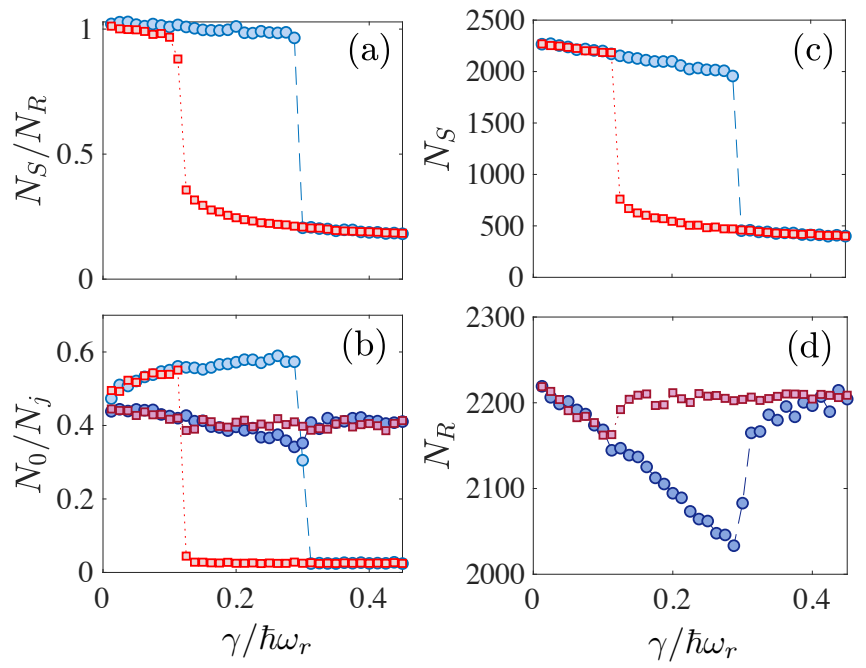

FIG. 9. Filling measures vs. $\gamma$ for the dynamical reservoir driving [Eqs. (28) and (29)], for parameters $J / \hbar \omega_{r}=0.3$ and $k_{B} T_{\text {eff }} / \hbar \omega_{r}=$ 30. (a) Relative filling for the lower branch (red squares) and upper branch (blue circles). (b) Condensate fraction of the system site (light markers) and one of the reservoirs (dark markers) for the lower branch (squares) and the upper branch (circles). (c,d) Atom number of the system (c) and one of the reservoirs (d) [markers are as in (b)].

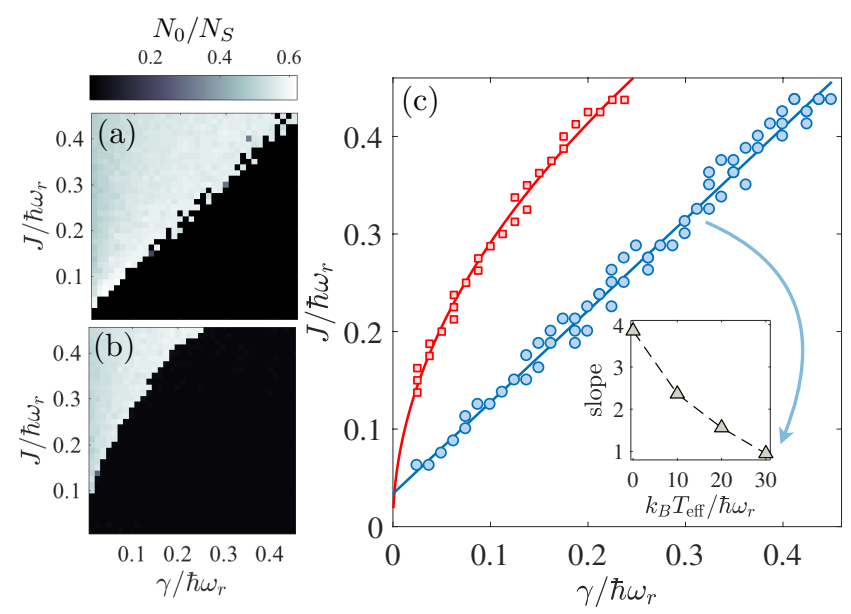

FIG. 10. Steady-state condensate fractions $N_{0} / N_{S}$ for the model including reservoir dynamics and backaction in the (a) full and (b) empty branches in the $J-\gamma$ plane. This figure can be directly compared with Fig. 5 which did not include reservoir dynamics. (c) The phase diagram determined by numerically extracting phase boundaries from (a) and (b). The upper and lower boundaries were extracted by detecting the boundary where the condensate fraction exceeded 0.7 , and the middle boundary shows where the it exceeded 0.4. The lines of best fit are $J=a \gamma+b, a=0.94 \pm 0.02, b=$ $0.034 \pm 0.004$ for the superfluid branch, and $J=a x^{b}, a=0.94 \pm 0.02$, $b=0.50 \pm 0.01$ for the lower branch. The inset in (c) shows the slope of the boundary for the superfluid branch obtained for a range of different effective temperatures. 


\section{DISCUSSION}

\section{A. Coherent Reservoir Model}

In our coherent reservoir model, we found that the superfluid branch exhibits little difference from the Josephson and driven-damped single-mode models. This is perhaps not surprising, as the reservoirs are perfectly coherent and determined by the ground state of $\mathcal{L}$. Although in terms of the harmonic oscillator basis many modes are relevant, from the perspective of eigenmodes of the GPE the system can still be reduced to a single mode.

The appearance of the quasicondensate in the lower branch, and its absence in the upper branch can be explained as follows. If the system site is full, the nonlinear interactions shift the energy such that particles from the reservoir sites can directly tunnel to the ground state of the system. However, when the system is empty the particles from the reservoir sites instead must tunnel into excited modes consistent with their energy. If the driving and dissipation occur on timescales comparable to the collision rate, then the system can thermalise and restore the condensate in the lowest-lying modes. However, if driving and dissipation operate on faster timescales than the collision rate, particles cannot reach the ground state before they are lost from the system.

The nonequilibrium phase diagram obtained from the coherent driving model has a remarkable qualitative resemblance to that obtained in the experiments of Labouvie et al. [4]. The partial condensation of this phase [Fig. 3(b)] is also consistent with their reported observation of the enhanced current in this region of the phase diagram, which suggested a partial superfluid transport. However, the distinct plateau in the filling fraction seen in Fig. 3(b) was not observed in the experiment. Finally, the critical slowing down on short timescales in our model (Fig. 6), which occur on timescales $\omega_{r} t \sim 10$, are broadly consistent with Labouvie et al. [4], where the timescale for slowing down was on the order of $\sim 10 \mathrm{~ms}$ to $\sim 20 \mathrm{~ms}$ and the trap frequency was $\omega_{r}=2 \pi \times 165 \mathrm{~Hz}$. The general form of the filling timescale in Fig. 6(b) also bears a clear qualitative resemblance to that observed in Labouvie et al. [4]. However, we note in our data that the largest timescale is between the quasicondensate and normal state phases, whereas the results of Labouvie et al. [4] seemingly suggest the largest timescale should be between the condensate and quasicondensate phases. The critical slowing down at long timescales (Fig. 7) presents a clear signature in the atom number fluctuations. Observation of this signature within a cold atom experiment may be more challenging, requiring several seconds of evolution time, but should nonetheless be within reach experimentally.

\section{B. Dynamical Reservoir Model - Backaction and Heating}

Our dynamical reservoir model is able to quantitatively reproduce the nonequilibrium phase boundary scalings $\gamma_{c} \approx J$ and $\gamma_{L B} \propto \sqrt{J}$ with an appropriate choice of effective temperature for the reservoirs. However, this model shows only tran- sient evidence of the quasicondensate phase (unless $T_{\text {eff }}=0$ ), and collapses to the normal phase at later times. It is important to note that all of the ingredients of finite temperature, independent reservoir dynamics, and back-action from the system site were all required to realise a reduction in the critical dissipation rate to $\gamma_{c} \approx J$. Neglecting any one of these features left the model with $\gamma_{c} \approx 4 J$. The reduction of $\gamma_{c}$ is clearly due to a rather complicated combination of effects that all cooperatively reduce the effective value of $J$, including dephasing of $\psi_{L}$ and $\psi_{R}$, a reduced Frank-Condon factor $\eta$ [see Eq. (9)] from reduced coherence, and a reduction of the population in the driving sites $\left(N_{R}\right.$ and $\left.N_{L}\right)$. Although the inset of Fig. 10(c) suggests that the effective temperature and dephasing are likely the dominant mechanisms that reduce $\gamma_{c} / J$, Fig 9(b) suggests that a counter-intuitive "parasitic condensation" phenomenon, whereby the coherence of the system is actually enhanced by the dissipation at the expense of the reservoir sites may also be a contributing factor.

\section{Future Work}

Although the main focus of this work has been modelling a specific experiment with an atomic BECs [4], our predictions could potentially be explored in a coherently pumped exciton-polariton superfluid. Specifically, a confining potential could be created through mechanical stresses [28], and it could be driven by a non-uniform pump laser [36] that is blue-detuned from the bottom of the trap. The intermediate quasicondensate regime presumably requires these features, as the modulational instabilities which give rise to pattern formation in the LLE seemingly do not occur in a uniform system described by Eq. (11) with $\beta>0$ (see e.g. Ref. [38]). In the exciton-polariton context, stronger driving and dissipation could be considered than is possible in the ultracold atom setting, which may uncover additional phase regions similar to the tri- and pentastability regions observed in Ref. [37] for a simple dimer system. Given the success of the c-field approach to this damped-driven system, our cfield approach may yield further insight into the results of a related experiment [11], where the phenomenon of negative differential conductivity (which may have use in atomtronic applications [49-51]) was observed in the undamped refilling dynamics of an initially depleted site $[9,51]$. By utilising a system with attractive interactions, or generalizing to multiple components, it may be possible to realize spontaneous pattern phenomena such as dissipative solitons [35], and Turing patterns [30, 52, 53], or Chimera states [40] in matter wave systems. It would also be of interest to extend the c-field model to include the full dynamics of all lattices sites and test the approximations made for the reservoirs in this paper, although this would be computationally challenging.

\section{CONCLUSION}

We have developed a c-field model of a multimode drivendissipative Josephson array as experimentally realised by 
Labouvie et al. [4]. The basic features of bistability and hysteresis observed in experiment can be understood qualitatively from a single-mode mean-field approximation to the model where it is analytically tractable. Numerically simulating the full multimode problem, we have found that the nonequilibrium phase diagram qualitatively matches the results of Labouvie et al. [4]. Importantly, our model suggests that the observed critical slowing down in the lower branch is due to the formation of a quasicondensate within a band of excited harmonic oscillator states, which are near-resonant with the driving frequency. While direct observation of the patterned quasicondensate phase may be difficult experimentally, a clear signature appears in the atom number fluctuations, in which we observe a distinct peak at the transition. Meanwhile, a reduced robustness of the superfluid branch is obtained in our model by incorporating effects of reduced coherence and the backaction of the system on the reservoir sites.

Finally, we have identified that that the Lugiato-Lefever equation, widely used in nonlinear optics and exciton- polariton contexts, can also describe nonequilibrium dynamics of driven-dissipative atomic superfluids. The degree of control available in ultracold atom systems allows for the tuning of parameters such as on-site energies, the sign of interactions, and could also introduce spin degrees of freedom. Further study of this system has the potential to realise as yet undiscovered nonequilibrium states of quantum matter.

\section{ACKNOWLEDGMENTS}

We are particularly grateful to Herwig Ott for discussions and feedback on drafts of this manuscript. We also thank Ewan Wright, Brian Anderson, Ashton Bradley, Samuel Begg, and Lewis Williamson for further discussions and input. This research was supported by the Australian Research Council Centre of Excellence in Future Low-Energy Electronics Technologies (project number CE170100039) and funded by the Australian Government.
[1] V. A. Brazhnyi, V. V. Konotop, V. M. Pérez-García, and H. Ott, Phys. Rev. Lett. 102, 144101 (2009).

[2] D. Witthaut, F. Trimborn, H. Hennig, G. Kordas, T. Geisel, and S. Wimberger, Phys. Rev. A 83, 063608 (2011).

[3] G. Barontini, R. Labouvie, F. Stubenrauch, A. Vogler, V. Guarrera, and H. Ott, Phys. Rev. Lett. 110, 035302 (2013).

[4] R. Labouvie, B. Santra, S. Heun, and H. Ott, Phys. Rev. Lett. 116, 235302 (2016).

[5] M. Biondi, G. Blatter, H. E. Türeci, and S. Schmidt, Phys. Rev. A 96, 043809 (2017).

[6] A. Schnell, D. Vorberg, R. Ketzmerick, and A. Eckardt, Phys. Rev. Lett. 119, 140602 (2017).

[7] C. V. Chianca and M. K. Olsen, Laser Physics 28, 015501 (2018).

[8] G. Kordas, S. Wimberger, and D. Witthaut, EPL (Europhysics Letters) 100, 30007 (2012).

[9] G. Kordas, D. Witthaut, and S. Wimberger, Annalen der Physik 527, 619 (2015).

[10] F. Verstraete, M. M. Wolf, and J. I. Cirac, Nature Physics 5, 633 (2009).

[11] R. Labouvie, B. Santra, S. Heun, S. Wimberger, and H. Ott, Phys. Rev. Lett. 115, 050601 (2015).

[12] H. Cartarius and G. Wunner, Phys. Rev. A 86, 013612 (2012).

[13] C. M. Bender and S. Boettcher, Phys. Rev. Lett. 80, 5243 (1998).

[14] H. J. Carmichael, Phys. Rev. X 5, 031028 (2015).

[15] N. R. Council, S. S. S. Committee, et al., Condensed-Matter and Materials Physics: The Science of the World Around Us, Vol. 4 (National Academies Press, 2008).

[16] F. Letscher, O. Thomas, T. Niederprüm, M. Fleischhauer, and H. Ott, Phys. Rev. X 7, 021020 (2017).

[17] D. Vorberg, W. Wustmann, R. Ketzmerick, and A. Eckardt, Phys. Rev. Lett. 111, 240405 (2013).

[18] T. Langen, R. Geiger, and J. Schmiedmayer, Annu. Rev. Condens. Matter Phys. 6, 201 (2015).

[19] T. Schweigler, V. Kasper, S. Erne, I. Mazets, B. Rauer, F. Cataldini, T. Langen, T. Gasenzer, J. Berges, and J. Schmiedmayer, Nature 545, 323 (2017).

[20] M. Pigneur, T. Berrada, M. Bonneau, T. Schumm, E. Demler, and J. Schmiedmayer, Phys. Rev. Lett. 120, 173601 (2018).

[21] S. Erne, R. Bücker, T. Gasenzer, J. Berges, and J. Schmiedmayer, Nature 563, 225 (2018).

[22] D. Husmann, M. Lebrat, S. Häusler, J.-P. Brantut, L. Corman, and T. Esslinger, Proceedings of the National Academy of Sciences 115, 8563 (2018).

[23] M. Prüfer, P. Kunkel, H. Strobel, S. Lannig, D. Linnemann, C.M. Schmied, J. Berges, T. Gasenzer, and M. K. Oberthaler, Nature 563, 217 (2018).

[24] A. Müllers, B. Santra, C. Baals, J. Jiang, J. Benary, R. Labouvie, D. A. Zezyulin, V. V. Konotop, and H. Ott, Science Advances 4 (2018), 10.1126/sciadv.aat6539.

[25] A. Trombettoni and A. Smerzi, Phys. Rev. Lett. 86, 2353 (2001).

[26] G. L. Alfimov, P. G. Kevrekidis, V. V. Konotop, and M. Salerno, Phys. Rev. E 66, 046608 (2002).

[27] A. Schnell, R. Ketzmerick, and A. Eckardt, Phys. Rev. E 97, 032136 (2018).

[28] I. Carusotto and C. Ciuti, Reviews of Modern Physics 85, 299 (2013).

[29] L. A. Lugiato and R. Lefever, Phys. Rev. Lett. 58, 2209 (1987).

[30] L. A. Lugiato, F. Prati, M. L. Gorodetsky, and T. J. Kippenberg, Philosophical Transactions of the Royal Society A: Mathematical, Physical and Engineering Sciences 376, 20180113 (2018).

[31] P. B. Blakie, A. S. Bradley, M. J. Davis, R. J. Ballagh, and C. W. Gardiner, Advances in Physics 57, 363 (2008).

[32] M. J. Davis, T. M. Wright, P. B. Blakie, A. S. Bradley, R. J. Ballagh, and C. W. Gardiner, in Quantum Gases: Finite Temperature and Non-Equilibrium Dynamics (World Scientific, 2013) pp. 163-175.

[33] I. Bloch, J. Dalibard, and W. Zwerger, Rev. Mod. Phys. 80, 885 (2008).

[34] I. V. Barashenkov and Y. S. Smirnov, Phys. Rev. E 54, 5707 (1996).

[35] M. A. Ferré, M. G. Clerc, S. Coulibally, R. G. Rojas, and M. Tlidi, The European Physical Journal D 71, 172 (2017).

[36] W. B. Cardoso, L. Salasnich, and B. A. Malomed, Scientific reports 7, 16876 (2017)

[37] S. R. K. Rodriguez, A. Amo, I. Sagnes, L. Le Gratiet, E. Ga- 
lopin, A. Lemaître, and J. Bloch, Nature Communications 7, 11887 (2016).

[38] M. Foss-Feig, P. Niroula, J. T. Young, M. Hafezi, A. V. Gorshkov, R. M. Wilson, and M. F. Maghrebi, Phys. Rev. A 95, 043826 (2017).

[39] S. S. Gavrilov, Phys. Rev. B 94, 195310 (2016).

[40] S. S. Gavrilov, Phys. Rev. Lett. 120, 033901 (2018).

[41] P. Comaron, G. Dagvadorj, A. Zamora, I. Carusotto, N. P. Proukakis, and M. H. Szymańska, Phys. Rev. Lett. 121, 095302 (2018).

[42] K. Dunnett, A. Ferrier, A. Zamora, G. Dagvadorj, and M. H. Szymańska, Phys. Rev. B 98, 165307 (2018).

[43] P. D. Drummond and D. F. Walls, Journal of Physics A: Mathematical and General 13, 725 (1980).

[44] J. P. Boyd, Chebyshev and Fourier spectral methods (Courier Corporation, 2001).

[45] S. J. Rooney, P. B. Blakie, and A. S. Bradley, Phys. Rev. E 89, 013302 (2014).

[46] G. R. Dennis, J. J. Hope, and M. T. Johnsson, Computer
Physics Communications 184, 201 (2013).

[47] See Supplemental Material at [URL will be inserted by publisher] for examples movies of the filling dynamics for the system in the normal state (NS), quasicondensate (QC), and superfluid (SF) phases.

[48] N. P. Proukakis, S. A. Gardiner, M. Davis, and M. Szymanska, Quantum Gases: Finite temperature and non-equilibrium dynamics, Vol. 1 (World Scientific, 2013).

[49] R. A. Pepino, J. Cooper, D. Z. Anderson, and M. J. Holland, Phys. Rev. Lett. 103, 140405 (2009).

[50] M. K. Olsen and J. F. Corney, Phys. Rev. A 94, 033605 (2016).

[51] D. Fischer and S. Wimberger, Annalen der Physik 529, 1600327 (2017), 1600327.

[52] W. J. Firth, A. J. Scroggie, G. S. McDonald, and L. A. Lugiato, Phys. Rev. A 46, R3609 (1992).

[53] J. B. Geddes, J. V. Moloney, E. M. Wright, and W. J. Firth, Optics Communications 111, 623 (1994). 


\section{Supplementary Files}

This is a list of supplementary files associated with this preprint. Click to download.

- QCJ0.35gamma0.7.mp4

- NSJ0.35gamma1.8.mp4

- SFJ0.35gamma0.65.mp4 